\title{
Cooperativas y responsabilidad social empresarial ${ }^{1}$
}

\author{
Alejandro Martínez Charterina \\ Catedrático de la Universidad de Deusto \\ Director del Instituto de Estudios Cooperativos de la Facultad de Derecho
}

Sumario: I. Eficiencia económica. II. Responsabilidad social empresarial. III. Identidad cooperativa. IV. Conclusión. V. Bibliografía.

Resumen: Las cooperativas naturalmente, es decir, por su propia identidad, asumen un compromiso de responsabilidad social en medio de un conjunto de empresas, mayoritariamente capitalistas, que pueden llegar a ese compromiso, en el mejor de los casos, cuando no interesadamente, de forma voluntaria.

Palabras clave: cooperativas, responsabilidad social, identidad cooperativa.

Abstract: Due to its specific nature, cooperative identity entails assuming a commitment to social responsibility in the midst of companies which are mostly capitalist. These latter companies may also embrace this commitment either in their own interest or voluntarily.

Key words: cooperatives, social responsibility, cooperative identity.

1 Texto basado en mi intervención sobre «La identidad cooperativa y la eficiencia económica» en el Encuentro de la Universidad Internacional Menéndez Pelayo titulado "La función social de la Sociedad Cooperativa en el siglo XXI», en Santander, el 10 de septiembre de 2013. 


\section{Eficiencia económica}

La ciencia de la economía, por lo menos desde los años treinta del pasado siglo en que Lionel Robbins ${ }^{2}$ la definió como el estudio de la conducta humana que trata de satisfacer necesidades ilimitadas con recursos escasos, nos invita a la eficiencia macroeconómica. Si no podemos satisfacer todas nuestras necesidades, entendidas de la forma más amplia posible, es decir, como equivalentes a deseos, que en consecuencia son ilimitadas, toda vez que los recursos de que disponemos son insuficientes para ello, una conducta racional nos invita a jerarquizar las necesidades y a aprovechar los recursos de forma eficiente, disponiendo el mínimo de recursos para cada necesidad lo que nos debe conducir a satisfacer el máximo de ellas y en consecuencia a alcanzar el máximo de nuestros deseos.

Al mismo tiempo contemplamos cómo en nuestras economías difícilmente se produce ese máximo aprovechamiento eficiente de los recursos en la medida en que mantenemos unas tasas de desempleo suficientemente, cuando no extremadamente, elevadas, o en la medida en que el equipo capital no se está utilizando plenamente sino en función de las horas de trabajo real de las empresas. Todo ello globalmente nos permite aspirar a una eficiencia económica mayor.

Esta eficiencia macroeconómica o del sistema en su conjunto a la que nos estamos refiriendo la podemos trasladar a las unidades económicas y plantear la eficiencia microeconómica o empresarial. La empresa, unidad de producción, actúa de forma eficiente cuando lleva a cabo su actividad con el máximo aprovechamiento de sus recursos. Y cuando hacemos de ello una traducción monetaria, esto significa que minimizamos los costes y consiguientemente maximizamos los beneficios.

\section{Responsabilidad social empresarial}

Las empresas capitalistas familiares, propias del proceso de industrialización, fueron desarrollando su dimensión de la mano de las sociedades por acciones, de modo que la segunda revolución industrial, a finales del siglo XIX, contaba ya con grandes sociedades e incluso con empresas internacionales.

Esta evolución continuó en el siglo xx, de forma que, a partir del final de la segunda guerra mundial, siguió creciendo la dimensión de las empresas, acompañada de la extensión de empresas multinacionales.

2 Lionel Robbins, An Essay on the Nature and Significance of Economic Science (1932). 
Estas multinacionales que primero serían norteamericanas, pero en adelante de muchos otros países, incluso de países emergentes, se fueron calificando de transnacionales para poner de manifiesto la ausencia de una nacionalidad de facto por su parte y en cambio la existencia de una transversalidad en cuanto a sus conductas que vendría a permitir el acomodo a las legislaciones más convenientes a sus propios intereses, generarían no pocas inquietudes en una sociedades que veía crecer su poder más allá incluso de los poderes públicos.

No es de extrañar que en esos años se empezaran a escuchar voces que trataban de situar estas empresas en el marco de una sociedad, cuyo medio ambiente se deterioraba por la acción de estas mismas empresas o por las condiciones de trabajo en determinados países en vías de desarrollo o por los escándalos de diverso orden, fugas de capitales, movimientos internacionales para evitar el pago de impuestos, paraísos fiscales, etc., de manera que durante la segunda mitad del siglo XX se han desarrollado teorías acerca del balance social, de los códigos de conducta, y de lo que hoy consideramos responsabilidad social de las empresas tratando de superar la idea de la empresa como un ente aislado que se plantea objetivos únicamente para dar satisfacción a sus propietarios-accionistas.

Es cierto que muchos de los estudios que se hicieron fueron dejando de tener interés en la medida en que no condujeron a ningún cambio, como la elaboración de códigos de conducta por y para las empresas multinacionales que, en ausencia de capacidad coercitiva, no tuvieron ninguna virtualidad.

Pero es cierto también que se fueron formando diversas posiciones doctrinales que al paso del tiempo han ido asentando posturas diferenciales que han ido llevando la idea de responsabilidad social empresarial (RSE) a la sociedad de nuestros días, con mayor o menor acogida, pero con una presencia indudable.

En el presente, si bien hay muchas variaciones y muchos acentos en el desarrollo doctrinal, se pueden identificar dos grandes grupos de teorías que revelan posiciones antagónicas acerca de los objetivos de la empresa y en consecuencia de la gestión que de las mismas debe realizarse.

En un lado están las teorías defensoras del Shareholder y en el otro las del Stakeholder ${ }^{3}$.

3 Están magnificamente recogidas en el contexto de los países anglosajones por Iñigo Zabala Ortiz de la Torre, «La pugna entre el shareholder primacy model y la stakeholder theory en la doctrina y práctica anglosajona. Estado de la cuestión» (2013). 
Las primeras, centradas en el shareholder, el accionista, arrancan del artículo de Milton Friedman de 1970 «La responsabilidad social de las empresas es aumentar sus beneficios ${ }^{4}$, y el razonamiento que lo sostiene nos dice que las empresas contribuyen a maximizar la eficiencia del sistema cuando maximizan sus beneficios, de forma que otras posibles responsabilidades conducirían necesariamente a una menor eficiencia en el uso de los recursos de la empresa 5 .

Las segundas consideran que la empresa no es un ente aislado, compuesto de accionistas, sino que, por el contrario, situada en la sociedad, lleva a cabo su función gracias a la participación de diversos agentes tanto internos como externos, además de los accionistas, los clientes, los proveedores, los trabajadores, los acreedores, la comunidad social..., es decir los stakeholders o «interesados» o «involucrados», que prestan a la empresa su trabajo, su capacidad financiera, su clientela, sus materias primas..., de modo que sin ellos la empresa no podría cumplir su función, y que, en consecuencia, merecen la atención de esta.

R. Edward Freeman ${ }^{6}$ comenzó esta teoría, que frente a la defensora de los accionistas que pone su mirada únicamente en la eficiencia, se fija en otros valores, sin rechazar la eficiencia, como la justicia, la confianza y la cooperación, señala Iñigo Zabala7, o llega a alcanzar su fundamento en el concepto de bien común, como considera Antonio Argandoña ${ }^{8}$.

Iñigo Zabala concluye, refiriéndose a la doctrina de los países anglosajones, "en la necesidad de proceder a una revisión en profundidad de cuál ha de ser el fin u objetivo de la sociedad», más aún en este tiempo de crisis financiera y retribuciones escandalosas, si bien el modelo de la teoría del stakeholder adolece de deficiencias que dificultan su plena puesta en marcha. "Tanto su falta de claridad, en lo que se refiere a la existencia de intereses distintos... como la inexistencia de mecanismos procesales... entendemos que será imposible la aplicación de esta teoría a una sociedad capitalista»?.

4 Milton Friedman, «The Social Responsability of Bussiness is to Increase its Profits», en The New York Times Magazine, del 13 de septiembre de 1970.

5 Puede verse Ignacio Aldeanueva Fernández y Carlos Ángel Benavides Velasco, «Responsabilidad social y ética. Aportaciones en el ámbito de los estudios sobre responsabilidad social» (2013), p. 167.

6 R. Edward Freeman, Strategic Management: a Stakeholder Approach (1984).

7 Iñigo Zabala Ortiz de la Torre, op. cit., p.118.

8 Lo que hace Antonio Argandoña en La teoría de los stakeholders y el bien común (1998).

9 Iñigo Zabala Ortiz de la Torre,op. cit., p.128. 
En la Unión Europea, como en el resto del mundo, la globalización y el desarrollo de los medios de comunicación han llevado a enfrentarse con una gestión de empresas más compleja, y a ello hay que sumar la crisis económica que pone en cuestión la validez del modelo capitalista.

En el mundo actual, dice José Antonio Villasante, «la empresa no puede ser concebida como una mera combinación eficiente de factores productivos para generar bienes y servicios... debe jugar además un papel social relevante como portadora de valores y generadora de comportamientos en una sociedad global donde cobran una nueva dimensión los intangibles como son la confianza y la reputación» ${ }^{10}$, volviendo a poner de nuevo en tela de juicio la validez de la consideración de la eficiencia económica sin ningún elemento corrector adicional.

En ese contexto de globalización y en un mundo que se siente amenazado por la abundancia de conductas irresponsables, el concepto de sostenibilidad deviene esencial como filtro a todo proyecto de carácter empresarial.

Es por ello por lo que desde el Consejo europeo de Lisboa del año 2000 se comenzó a definir el concepto de Responsabilidad Social Empresarial (RSE), o Responsabilidad Social Corporativa (RSC), para introducir las ideas de desarrollo social y de sostenibilidad e incorporar la consideración social y medioambiental junto a la estrictamente económica en la valoración empresarial: «En Lisboa se intentó combinar un fuerte desarrollo social con la competitividad empresarial. La RSC podría contribuir a un mejor desempeño de la actividad empresarial desde el punto de vista medioambiental y a una mayor cohesión social»»11.

Desde entonces tanto la Comisión como el Parlamento Europeo han realizado una serie de acciones y producido sin pausa una documentación sobre la materia12:

- En 2001:

- El primer documento oficial de la Comisión sobre RSC, el Libro Verde: «Fomentar un marco europeo para la Responsabilidad Social de las Empresas».

10 José Antonio Villasante en el Prólogo a la obra de José Antonio Galán y Antonio Sáenz de Miera (editores), Reflexiones sobre la responsabilidad social corporativa en el siglo XXI (2012), p. 9.

11 Dominique Bé, «Pasado y presente de la responsabilidad social de la empresa» (2004), p. 39.

12 Puede verse Antoni Gelonch-Viladegut, «La RSC en Europa: retos y oportunidades» (2012). 
- En 2002:

- Comunicación de la Comisión »La Responsabilidad Social de las Empresas: Una contribución empresarial al desarrollo sostenible».

- Se constituyó el Foro Multistakeholders en RSC.

- Primera resolución del Parlamento aprobando el Foro e invitando a realizar informes de RSC.

-En 2003:

- Segunda resolución del Parlamento sobre RSC, trata de la importancia del papel de la economía para el desarrollo sostenible.

- En 2004:

- Informe del Foro Multistokholders, invitando a la realización de un marco legal de la RSC.

- En 2005:

- Documento de la Comisión «Trabajando juntos para el crecimiento y el empleo. Un Nuevo comienzo para la Estrategia de Lisboa».

- En 2006:

- Segundo comunicación de la Comisión sobre RSC «Poner en práctica la asociación para el crecimiento y el empleo: Hacer de Europa un polo de excelencia de la Responsabilidad Social de las Empresas».

- En 2007:

- Nueva resolución del Parlamento aprobando el «Informe sobre la Responsabilidad Social de las Empresas: Una nueva asociación», conocido como Informe Howitt, que fue el ponente del mismo.

- Informe de la Comisión sobre RSC para pequeñas y medianas empresas «Oportunidades y responsabilidad. Cómo ayudar a un mayor número de pequeñas empresas a integrar las cuestiones sociales y medioambientales en sus actividades».

- En 2008:

- Informe anual europeo de Competitividad de la Comisión, con un apartado sobre el impacto positivo de la RSC en la competitividad empresarial y la necesidad de fomentarla en tiempo de crisis. 
- En 2009:

- Documento de trabajo de la Comisión «Consulta sobre la futura Estrategia Europa 2020».

- En 2010:

- Comunicación de la Comisión «Comunicación de la Comisión Europa 2020: Una estrategia para un crecimiento inteligente, sensible e integrador».

- En 2011:

- Comunicación de la Comisión «Estrategia renovada de la UE para 2011-2014 sobre la responsabilidad social de las empresas».

De este último documento podemos extraer algunas ideas que revelan el alcance que tiene para la UE este tema de la responsabilidad social de las empresas.

Recoge esta Comunicación en primer lugar el concepto de responsabilidad social de las empresas con el que la Comisión Europea ha venido trabajando desde el año 2001: se trata de «la integración voluntaria, por parte de las empresas, de las preocupaciones sociales y medioambientales en sus operaciones comerciales y sus relaciones con sus interlocutores» ${ }^{13}$. Por consiguiente la responsabilidad social de las empresas supone la voluntariedad, al margen de otras obligaciones legales.

Considera la Comisión que la responsabilidad social de las empresas resulta beneficiosa tanto para las mismas empresas que la asumen, a través de sus relaciones con los stakeholders, en términos de competitividad, expectativas de futuro y confianza que habrá de conducir a un mejor entorno de innovación y crecimiento, como para el conjunto de la sociedad a través del desarrollo sostenible y de una economía de mercado altamente competitiva.

En el momento presente la crisis económica y sus consecuencias han hecho perder confianza de la sociedad en sus empresas y es, en consecuencia, más importante, insistir en la responsabilidad social ${ }^{14}$.

Se ha avanzado en esta primera década del siglo XXI, pero quedan retos pendientes.

13 Comunicación de la Comisión Europea, Estrategia renovada de la UE para 2011 2014 sobre la responsabilidad social de las empresas (2011), p. 4.

14 Véase Comunicación de la Comisión Europea, Estrategia renovada..., op. cit., pp. 4-5. 
De aquí que se introduzca una nueva definición: la responsabilidad social de las empresas es «la responsabilidad de las empresas por su impacto en la sociedad $\rangle^{15}$. Previo a la responsabilidad social es el respeto a la legislación vigente y el cumplimiento de los convenios colectivos. Pero la responsabilidad social exige la integración de las preocupaciones sociales, medioambientales y éticas, el respeto de los derechos humanos y la consideración de los consumidores en la actividad y en la estrategia empresarial.

Recomienda a las empresas que quieren avanzar en la responsabilidad social la orientación de los principios y directrices internacionales en la materia que constituyen el marco para la RSE, citando expresamente «las Líneas Directrices de la OCDE para las empresas multinacionales, los diez principios de la iniciativa del Pacto Mundial de las Naciones Unidas, la norma de orientación ISO 26000 sobre responsabilidad social, la Declaración tripartita de principios sobre las empresas multinacionales y la politica social de la OIT y los Principios Rectores de las Naciones Unidas sobre Empresas y Derechos Humanos» ${ }^{16}$.

Finalmente se propone un Programa de Acción para el periodo 2011-2014 en el que se contienen unos compromisos de la Comisión en términos de estudio, informe y acciones facilitadoras, y una serie de sugerencias para las empresas, los Estados miembros y otros grupos interesados ${ }^{17}$.

\section{Identidad cooperativa}

Previo al estudio de la identidad cooperativa resulta importante señalar que la cooperativa es una empresa, lo que no siempre ha sido admitido por una parte de la doctrina que ha considerado como empresa solamente a la empresa capitalista, es decir, a aquella empresa en la que el empresario es justamente el que aporta el capital.

En esta empresa capitalista el empresario recibe el dividendo que es la suma del interés del capital y el beneficio empresarial, y como el beneficio es variable, también lo es el dividendo. Las decisiones se toman en función del capital aportado, cada acción tiene un voto, y el dividendo se reparte también en función del capital, una cantidad por acción.

15 Comunicación de la Comisión Europea, Estrategia renovada..., op. cit., p. 7.

16 Comunicación de la Comisión Europea, Estrategia renovada..., op. cit., p. 8.

17 Puede verse Comunicación de la Comisión Europea, Estrategia renovada..., op. cit., pp. 10-18. 
En la cooperativa, por el contrario, el empresario puede ser el cliente, lo que sucede por ejemplo en las cooperativas de consumidores, el proveedor, como en las bodegas cooperativas en las que los agricultores entregan la uva para que la empresa produzca el vino y lo comercialice, los trabajadores que son los socios de la cooperativa de trabajo asociado ${ }^{18} \ldots$

En estas cooperativas el capital lo ponen los socios porque no encuentran a un capitalista que esté dispuesto a ponerlo a cambio de una retribución, interés, fijo y al margen del beneficio. Ahora bien las decisiones se toman de forma democrática, cada persona tiene un voto con independencia de su aportación de capital, y el beneficio se reparte, en su caso, de forma distinta a la proporcional en función del capital aportado. Así en una cooperativa de consumidores el beneficio se distribuirá según el consumo realizado, en una formada por los proveedores, en función de la cantidad y calidad de lo entregado, en una de trabajadores, en función del trabajo realizado...

Las cooperativas, desde el origen del cooperativismo moderno, es decir, desde la fundación de la cooperativa de Rochadale en 1844, adaptan su comportamiento al cumplimiento de unos principios. Estos primeros principios de Rochdale han ido actualizándose por la Alianza Cooperativa Internacional y en el Congreso centenario de su fundación, en 1995, aprobó una Declaración sobre la identidad cooperativa.

Esa declaración tiene tres partes, la definición, los valores y los principios, que conforman la identidad cooperativa.

Para la Alianza Cooperativa Internacional una cooperativa es una «asociación autónoma de personas que se han unido de forma voluntaria para satisfacer sus necesidades y aspiraciones económicas, sociales y culturales en común mediante una empresa de propiedad conjunta y de gestión democrática» ${ }^{19}$.

Esta definición sigue la idea que popularizó Georges Fauquet ${ }^{20}$ al considerar que en la cooperativa confluyen dos elementos inseparables, el social y el económico. De este modo la cooperativa es a la vez una asociación de personas unidas por su deseo de satisfacer unas necesidades o alcanzar unas aspiraciones y una empresa de la que son propietarias esas mismas personas que la gestionan a través de la cual hacen realidad sus deseos y aspiraciones.

18 Puede verse en este sentido el esquema y la descripción que realiza Georges Lasserre, «Signification économique et morale des règles de Rochdale» (1967), p. 322.

19 ICA, Declaración de la Alianza Cooperativa Internacional sobre la identidad cooperativa (1996), p. 17.

20 Vease Georges Fauquet, «Le secteur coopératif», en Oeuvres (1965), pp. 39-40. 
Resulta así esencial la conexión de la empresa con el grupo de personas "que la crea y le proporciona su ideología, su capital, su fuerza de trabajo y su clientela» ${ }^{21}$.

En nuestro entorno próximo José M. ${ }^{a}$ Arizmendiarreta, inspirador de las cooperativas de Mondrágón, recogía la idea para estas cooperativas diciendo lo siguiente: "La experiencia ha correspondido a una concepción en la que lo social debe acreditarse por lo económico, no menos que lo económico debe autentificarse por lo social» ${ }^{22}$.

La Decalaración de la $\mathrm{ACI}$ sobre la Identidad Cooperativa considera que la actividad de las cooperativas tiene su base en unos valores o aspiraciones como son «la autoayuda, la autoresponsabilidad, la democracia, la igualdad, la equidad y la solidaridad», y que los socios exhiben unos valores éticos de «honestidad, transparencia, responsabilidad $y$ vocación sociales» ${ }^{23}$.

Por fin los principios cooperativos que son «pautas mediante las cuales las cooperativas ponen en práctica sus valores» y a los que estas conforman su actividad. Son los siguientes 24 :

1. Adhesión voluntaria y abierta.

2. Gestión democrática por parte de los socios.

3. Participación económica de los socios.

4. Autonomía e independencia.

5. Educación, formación e información.

6. Cooperación entre cooperativas.

7. Interés por la comunidad.

El primer principio es el conocido como de puerta abierta, que permite entrar y salir de la cooperativa de forma voluntaria y sin discriminación de sexo, social, racial, política o religiosa.

El segundo confirma a la cooperativa como una empresa que se gestiona de forma democrática, de manera que cada socio, cada persona, tiene un voto, con independencia de cualquier otra consideración.

El tercer principio, de participación económica de los socios, recoge la contribución equitativa de los socios al capital de sus cooperativas y

21 Alejandro Martínez Charterina, «La cooperativa como empresa y los principios cooperativos tras el congreso centenario de la A.C.I. de Manchester», en Evolución del escenario económico (1996), p. 208.

22 José María Arizmendiarrieta, «La experiencia cooperativa de Mondragón», en Homenaje a Del Arco: Del arco treinta años de vida cooperativa (1973), p. 68.

23 ICA, Declaración de la Alianza Cooperativa Internacional sobre la identidad cooperativa (1996), p. 17.

24 Puede verse ICA, Declaración de la Alianza Cooperativa Internacional sobre la identidad cooperativa (1996), pp. 17-19. 
la compensación limitada si la hubiere (interés limitado al capital), así como el destino del beneficio al fortalecimiento de la cooperativa a través de las reservas, al reparto en función de las operaciones de los socios con la cooperativa, y al apoyo de otras actividades aprobadas por los socios.

El cuarto principio se fija en las relaciones con otras organizaciones, hoy especialmente para reforzar sus capacidades de financiación, lo que debe hacerse asegurando el control democrático de los socios y la autonomía de la cooperativa.

El quinto constituye la que se conoce como regla de oro de la cooperación, es decir, la educación y formación de socios, directivos y empleados, para su capacitación profesional y perfeccionamiento personal, así como la información a la sociedad, especialmente a los jóvenes y a los líderes de opinión acerca de los beneficios de la cooperación.

El sexto principio, de intercooperación, considera la necesidad de fortalecer desde el punto de vista representativo y desde el económico el movimiento cooperativo, a base de la colaboración de unas cooperativas con otras a todos los niveles, local, nacional e internacional.

Por fin el sétimo principio muestra el interés por la comunidad ordenando la aportación de la cooperativa al desarrollo sostenible de la misma.

Refiriéndose a los principios cooperativos dice el Informe que acompaña a la Declaración de la Alianza Cooperativa Internacional sobre la Identidad Cooperativa que «son las cualidades esenciales que hacen que los cooperativistas sean eficaces, las cooperativas diferentes, y el movimiento cooperativo valioso» 25 .

Las cooperativas lo son precisamente en cuanto acomodan su conducta a esta identidad. Es frecuente que las leyes de cooperativas remitan a los principios de la Alianza al proceder a su definición. Así en la Ley General26, que en su artículo 1. ${ }^{\circ}$ dice "La cooperativa es una sociedad constituida por personas que se asocian, en régimen de libre adhesión y baja voluntaria, para la realización de actividades empresariales, encaminadas a satisfacer sus necesidades y aspiraciones económicas y sociales, con estructura y funcionamiento democrático, conforme a los principios formulados por la alianza cooperativa internacional en los términos resultantes de la presente ley».

25 ICA, Declaración de la Alianza Cooperativa Internacional sobre la identidad cooperativa (1996), p. 67. 1999)

26 Ley 27/1999, de 16 de julio, de cooperativas (BOE n. ${ }^{\circ} 170$, de 17 de julio de 
El artículo primero de la Ley de Cooperativas de Euskadi27, que es anterior a la aprobación por parte de la Alianza Cooperativa Internacional de la Declaración sobre la Identidad Cooperativa, procede del mismo modo: «La cooperativa es aquella sociedad que desarrolla una empresa que tiene por objeto prioritario la promoción de las actividades económicas y sociales de sus miembros y la satisfacción de sus necesidades con la participación activa de los mismos, observando los principios del cooperativismo y atendiendo a la comunidad de su entorno.

La cooperativa deberá ajustar su estructura y funcionamiento a los principios cooperativos, que serán aplicados en el marco de la presente ley. Dentro de ésta actuará con plena autonomía e independencia respecto de cualesquiera organizaciones y entidades, públicas o privadas».

La cooperativa «no tiene nada que ver con el principio de máximo lucro sino con el de la satisfacción óptima de las necesidades» ${ }^{28}$.

Y en su razón de ser no se queda en sí misma sino que «tiende a conquistar y transformar la organización económica y social del mundo», frase con la que Paul Lambert introduce lo que llama «aspiración de conquista» como un rasgo fundamental de las cooperativas desde la perspectiva de la Alianza Cooperativa Internacional29.

De este modo, en consecuencia, las cooperativas por su propia naturaleza asumen el compromiso de la responsabilidad social porque acomodan su funcionamiento a la búsqueda de unos valores y la observación de unos principios que, más allá del balance económico, se refieren al social y medioambiental.

Precisamente por ello Iñigo Zabala finaliza la Conclusión del artículo al que hecho referencia anteriormente con las siguientes palabras: «En tanto en cuanto la doctrina no sea capaz de idear un sistema de evaluación y de asignación de cuotas entre los distintos tipos de interesados de cada sociedad (lo cual vemos como una misión imposible), entendemos que será imposible la aplicación de esta teoría a una sociedad capitalista. Ante esta imposibilidad, y si queremos realmente una forma social justa, que mantenga el principio liberal constitutivo y de funcionamiento empresarial, solamente nos quedará acudir a la Sociedad Cooperativa»30.

27 Ley 4/1993, de 24 de junio, de Cooperativas de Euskadi (BOPV n. ${ }^{\circ} 135$, de 19 de julio de 1993).

28 Paul Lambert, La doctrina cooperativa (1970), p. 273.

29 Paul Lambert, O.C., (1970), p. 272.

30 Iñigo Zabala Ortiz de la Torre, op. cit., p.128. 


\section{Conclusión}

Cabe decir en conclusión que en el debate entre la consideración de que la empresa debe centrarse en sus accionistas y mirar, en consecuencia, por el beneficio, contribuyendo de ese modo a la eficiencia económica, y la introducción de unos objetivos de sostenibilidad que junto al balance económico introducen el social y medioambiental, ampliando las obligaciones de la empresa más allá de sus accionistas a los interesados o involucrados internos y externos a la misma, existe y que, al menos a nivel de debate, las posiciones favorables a las teorías de los stakeholders ganan posiciones, sobre todo en la Unión Europea.

Dicho esto, debe considerarse también el carácter voluntario de la Responsabilidad Social de las Empresas.

La mayor parte de las empresas, que son sociedades capitalistas, que se acercan a la RSE, lo hacen, por tanto de forma voluntaria, y algunas de ellas tal vez estén esperando obtener un resultado económico mejor, tanto o más que contribuir a la sostenibilidad del sistema, la obtención de unos resultados sociales y el cuidado del medio.

Es tal sentido señalaba Dante Cracogna que «la tentación de vender imagen no debe seducir a las cooperativas, pero no debe descuidarse tampoco que otros aprovechen una tradicional lentitud y reticencia de las cooperativas a mostrar sus propias realizaciones para ganarles la delantera ante la consideración pública»31.

Y es que las cooperativas, desde la doble consideración de grupo social y económico que forma su concepto, se unen el vivir y el convivir. El «vivir con» implica la existencia de valores sociales, que es tanto como tamizar la eficiencia a través del filtro de los valores.

Desde los valores y principios de su identidad, especialmente los valores de responsabilidad social y de vocación social a que son llamados los socios, y el principio séptimo de interés por la comunidad que dirige la actividad cooperativizada al desarrollo sostenible de la misma, las cooperativas asumen la RSE por su misma naturaleza.

\section{Bibliografía}

ALDEANUEVA FERNÁNDEZ, Ignacio y BENAVIDES VELASCO, Carlos Ángel. «Responsabilidad social y ética. Aportaciones en el ámbito de los estudios sobre responsabilidad social», en Boletín de Estudios Económicos, n. ${ }^{\circ} 208$. Universidad Comercial de Deusto. Bilbao, 2013, ps. 165 -181.

31 Dante Cracogna, «La eficiencia social de las cooperativas y su evolución» (1981), p. 125. 
ARGANDOÑA, Antonio. La teoría de los stakeholders y el bien común. Documento de investigación n. ${ }^{\circ}$ 355. IESE - Universidad de Navarra, Barcelona, 1998. www.iese.edu/research/pdfs/DI-0355.pdf

ARIZMENDIARRIETA, José María. "La experiencia cooperativa de Mondragón», en obra colectiva, Homenaje a Del Arco: Del arco treinta años de vida cooperativa, CENEC, Zaragoza, 1973, ps. 65-73.

$B E$, Dominique. «Pasado y presente de la responsabilidad social de la empresa», en Miguel Osorio (comp.), La nueva empresa. Responsabilidad Social Corporativa. Voz de Papel, Madrid, 2004, ps. 37-46.

Comisión Europea. Estrategia renovada de la UE para 2011-2014 sobre la responsabilidad social de las empresas, Bruselas, 25.10.2011, en www.observatorio.org/images/stories/audio/publicaciones/con2011_RSC.pdf

CRACOGNA, Dante. "La eficiencia social de las cooperativas y su evolución», en Masón, Rubén et al., La eficiencia de las cooperativas. INTERCOOP, Buenos Aires, 1981, pp. 103-125.

FAUQUET, Georges. "Le secteur coopératif», en Oeuvres, Publications de I'Institut des Etudes Coopératives, Paris, 1965, pp. 21-127.

FREEMAN, R. Edward. Strategic Management: a Stakeholder Approach, Pitman, Boston, 1984.

GELONCH-VILADEGUT, Antoni. "La RSC en Europa: retos y oportunidades», en José Ignacio Galán y Antonio Sáez de Miera (Editores), Reflexiones sobre la responsabilidad social corporativa en el siglo XXI. Ediciones Universidad de Salamanca, Salamanca, 2012, pp. 169-193.

ICA. Declaración de la Alianza Cooperativa Internacional sobre la identidad cooperativa; Consejo Superior de Cooperativas de Euskadi, Vitoria-Gasteiz, 1996.

LAMBERT, Paul. La doctrina cooperativa. 3. ${ }^{a}$ edición. Intercoop, Buenos Aires, 1970.

LASSERRE, Georges. «Signification économique et morale des règles de Rochdale», en Les Colloques de l'Institut des Etudes Coopératives, III: Les príncipes coopératifs, hier, aujourd'hui, demain. Editions de l'Institut des Etudes Coopératives, Paris, 1967, pp. 279-323.

MARTíNEZ CHARTERINA, Alejandro. "La cooperativa como empresa y los principios cooperativos tras el congreso centenario de la A.C.I. de Manchester», en obra colectiva, Evolución del escenario económico, Universidad de Deusto, Bilbao, 1996, pp. 207-226.

ROBBINS, Lionel. An Essay on the Nature and Significance of Economic Science, Macmillan \& Co., London, 1932.

VILLASANTE, José Antonio. Prólogo a la obra de José Antonio Galán y Antonio Sáenz de Miera (editores), Reflexiones sobre la responsabilidad social corporativa en el siglo XXI, 2012, pp. 9-12.

ZABALA ORTIZ DE LA TORRE, Iñigo. "La pugna entre el shareholder primacy model y la stakeholder theory en la doctrina y práctica anglosajona. Estado de la cuestión», en Deusto Estudios Cooperativos, n. ${ }^{\circ}$ 2, Facultad de Derecho - Universidad de Deusto, Bilbao, 2013, pp. 103-132. 